\title{
Adjustable Speed Surface Subdivision
}

\author{
K. Karčiauskas and J. Peters \\ Department of Mathematics and Informatics, Vilnius University \\ Dept C.I.S.E., University of Florida
}

\begin{abstract}
We introduce a non-uniform subdivision algorithm that partitions the neighborhood of an extraordinary point in the ratio $\sigma: 1-\sigma$, where $\sigma \in(0,1)$. We call $\sigma$ the speed of the non-uniform subdivision and verify $C^{1}$ continuity of the limit surface. For $\sigma=1 / 2$, the Catmull-Clark limit surface is recovered. Other speeds are useful to vary the relative width of the polynomial spline rings generated from extraordinary nodes.
\end{abstract}

Key words: non-uniform, subdivision, adjustable speed,

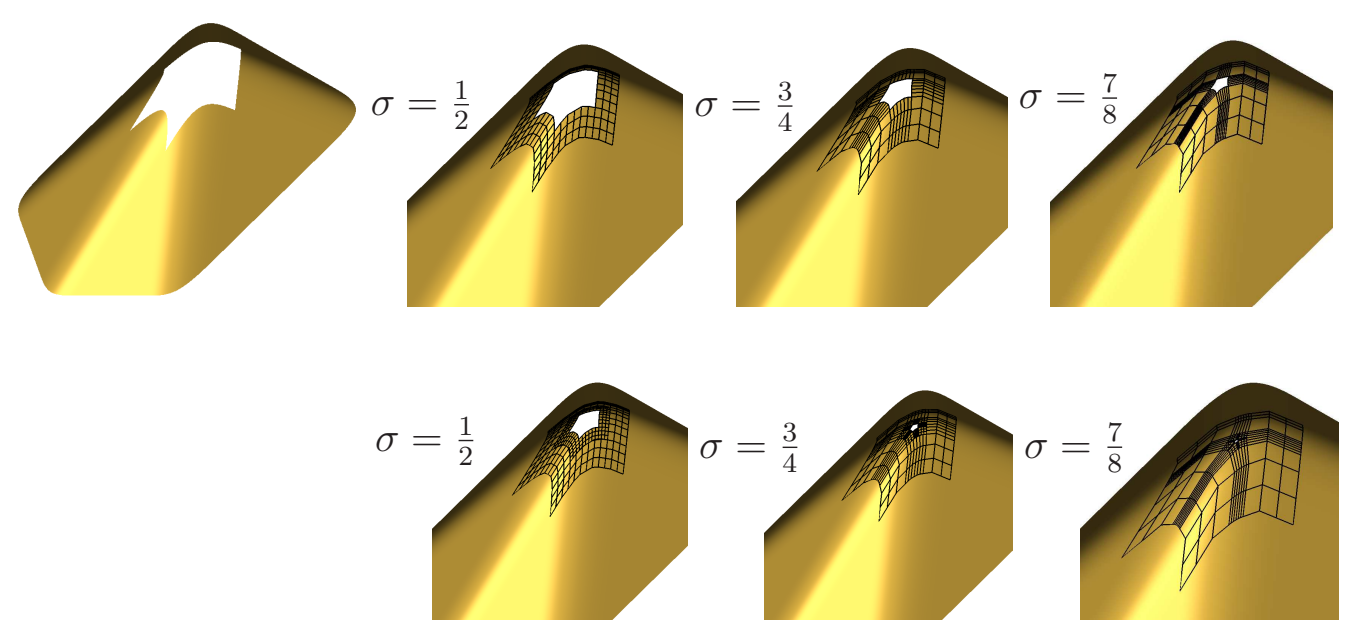

Fig. 1. (left) Input with gap and (right three columns) fillings with (top) one, respectively (bottom) two adjustable speed subdivision steps.

Email address: jorg@cise.ufl.edu, kestutis.karciauskas@mif.vu. It (K. Karčiauskas and J. Peters).

URL: http://www. cise.ufl. edu/ jorg (K. Karčiauskas and J. Peters). 


\section{Introduction}

To be able to control and adjust, possibly repeatedly, the relative width of the polynomial surface rings generated by a Catmull-Clark-like subdivision algorithm (see Figure 1), we derive rules for a new class of non-uniform surface subdivision algorithms. The resulting family of algorithms has properties akin to Catmull-Clark subdivision and can in particular generate the limit surface of Catmull-Clark subdivision. Figure 8 shows an application where fast filling of a $n$-sided surface hole is advantageous. In general, the fill speed must depend on the application since the surface quality deteriorates for more rapid fills. We have also used the characteristic rings of adjustable speed subdivision as concentric tessellation map of guided subdivision surfaces [KP07b], where the speed controls the parameterization rather than the shape (see Figure 9). The theory is advanced by the paper in that it explores a non-traditional approach that allows analyzing the resulting non-uniform subdivision surface. Figure 2 illustrates adjustable speed subdivision in one vari-

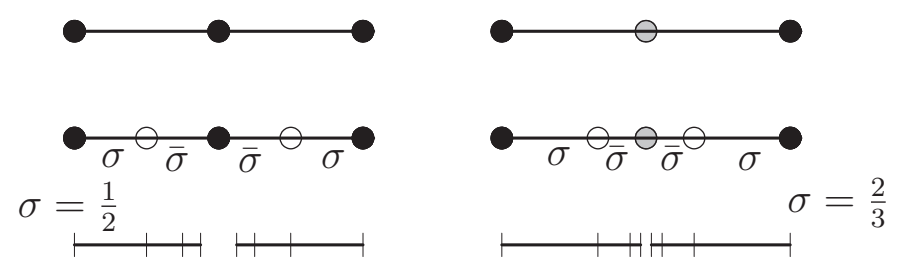

Fig. 2. Univariate localized subdivision near a (central) point, (left) in the standard setting and (right) adjustable speed subdivision (local truncated geometric progression per half space) with speed $\sigma=2 / 3$. (top two) control point refinement, (bottom) knot spacing.

able. Instead of subdividing uniformly, we locally subdivide in a ratio $\sigma: \bar{\sigma}$ where $\sigma \in(0,1)$ and $\bar{\sigma}:=1-\sigma$. We call $\sigma$ the speed of the non-uniform subdivision. Since splines are invariant when scaling all knots uniformly or when translating the knot sequence, only the knot spacing is in the following of interest.
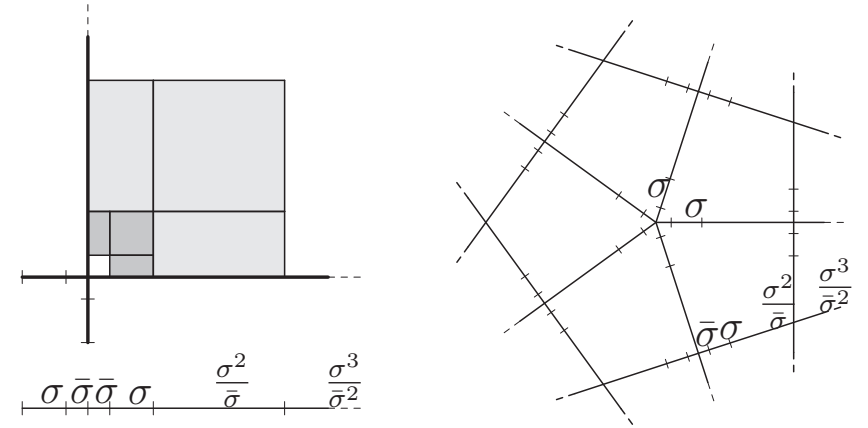

Fig. 3. Bivariate adjustable speed subdivision. (left)(bottom) Geometrically increasing knot spacing $s=\left\{\bar{\sigma}, \sigma, \frac{\sigma^{2}}{\bar{\sigma}}\right\}$ tensored (top) for adjustable speed subdivision. (right) Consistent knot spacings around an extraordinary node of valence $n=5$. The corresponding surface is a sequence of $C^{2}$-connected surface rings of degree bi-3.

Tensoring this subdivision, as shown in Figure 3, we obtain an adjustable speed 
subdivision that is well-defined except near vertices with $n \neq 4$ neighbors. Adding rules for these extraordinary cases, we obtain a family of subdivision algorithms generating a sequence of polynomial surface rings of degree bi-3 with contraction speed $\sigma \in(0,1)$. After reviewing the related literature, we derive the rules for adjustable speed subdivision in Section 2. In Section 3, we verify properties of adjustable speed subdivision and in Section 4, we discuss some applications.

\subsection{Prior Work}

With few exceptions, polynomial subdivision surface algorithms generalize uniform (box-)spline subdivision [BHR93]. In the univariate case, standard B-spline knot insertion yields a stable, local non-uniform refinement of the control polygon, one knot at a time. As an alternative to the Oslo algorithm [CLR80], recently new factorizations of non-uniform B-spline subdivision have been proposed [CDS07,CDS08,SG08]. Earlier, Goldman and Warren [GW93] extended uniform subdivision of curves to knot intervals in geometric progression. Knot intervals of adjustable speed subdivision also have geometric progression, but since the neighborhood of an extraordinary node is combinatorially different, we cannot directly apply the rules in [GW93] even in the univariate case. When the univariate refinement is tensored and applied to tensor-product splines, the knot intervals must be carried over to parallel iso-curves. In practice, knots are inserted to obtain a least superset of the iso-knot intervals. By contrast, Non-uniform Recursive Subdivision Surfaces, proposed by Sederberg et al. [SZSS98], allow freely assigning knot intervals to every edge of the control net. To enable an analysis of this approach, [SZBN03] restricts this freedom by requiring that opposing edges of each foursided face have identical knot intervals. In either case, subdivision in the regular part surrounding an extraordinary node halves each knot interval. The feature-filled ESubs approach of Mueller [MRF06] allows analyzing extraordinary neighborhoods by switching to Catmull-Clark subdivision rules near extraordinary nodes. No such switch to uniform subdivision is necessary to fully analyze adjustable speed subdivision.

\section{An adjustable speed subdivision}

Since our knot spacing will be non-uniform, we can not hope to build on box-spline subdivision which is inherently uniform; and since the structure at the central point differs from that of the tensor-product mesh, we cannot just insert a knot to add $\frac{\bar{\sigma}^{2}}{\sigma}$ as an additional knot interval. Instead, we derive the rules by prolongation, namely surface ring extension in BB (Bernstein-Bézier) form. This is illustrated in Figure 4 and explained below. We use two different indexing strategies for input and output. The indexing of the input $\mathbf{p}$ is natural for representing patches of the surface ring. 


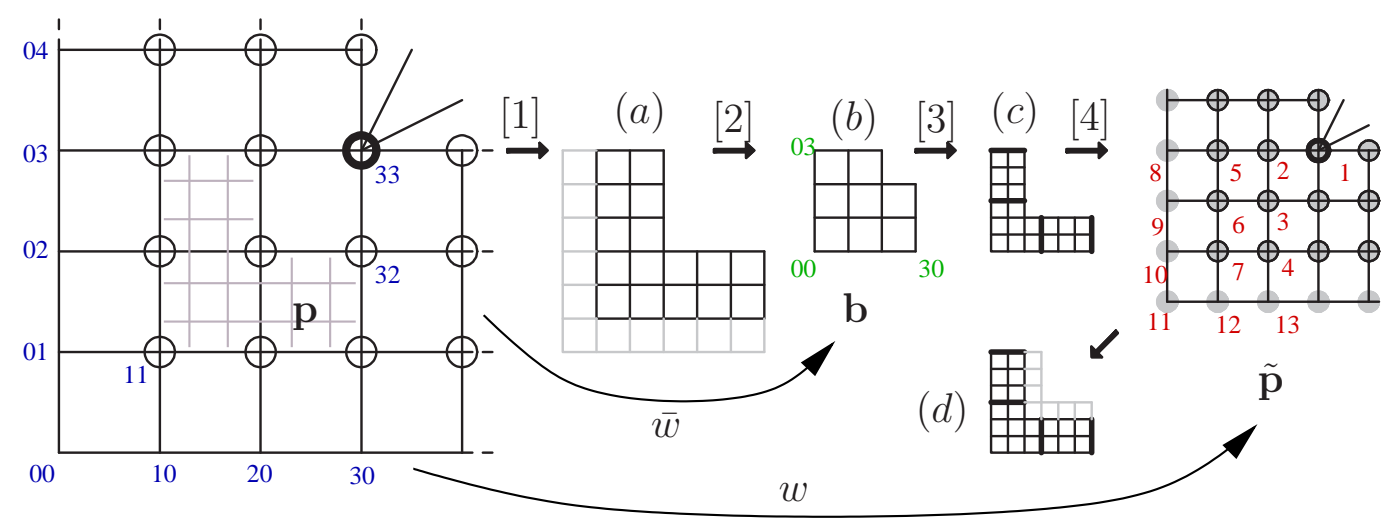

Fig. 4. Combinatorics and Indexing for deriving the subdivision rules. One sector of the input net $\mathbf{p}$ is converted to the output net $\tilde{\mathbf{p}}$ via the Bernstein-Bézier (BB-)representation $\mathbf{b}$. The knot spacings associated with $\mathbf{p}$ and $\tilde{\mathbf{p}}$ are in geometric progression.

The indexing of the output $\tilde{\mathbf{p}}$ is commonly used to analyze subdivision.

\section{Derivation of the Subdivision Rules (2)}

Input: A net $\mathbf{p}$ (Figure 4, left), whose points $\mathbf{p}^{i j}$ are coefficients of a bi-3 spline with a non-uniform knot spacing $\{s\}$ according to Figure 3.

Output: The refined net $\tilde{\mathbf{p}}$ (Figure 4, right) representing coefficients of a bi-3 spline with knot spacing $\left\{\frac{\bar{\sigma}^{2}}{\sigma}, s\right\}$.

Step [1] Convert $\mathbf{p}$ to bi-3 BB form $(a)$.

Step [2] Extend $(a) C^{2}$ inwards to yield coefficients $(b)$.

Step [3] Subdivide $(b)$ with ratio $\sigma: \bar{\sigma}$ to yield $(c)$.

Step [4] Revert (c) to $\tilde{\mathbf{p}}$. The extraordinary node is defined by the stencil of Figure 5 ,left, applied to the input net $\mathbf{p}$ with the choice

$$
\beta:=\frac{18 \sigma \bar{\sigma}\left(\sigma^{2}+\bar{\sigma}\right)}{9+(n-4)(\bar{\sigma}+1)^{2}}, \quad \gamma:=\frac{9 \sigma^{2} \bar{\sigma}^{2}}{9+(n-4)(\bar{\sigma}+1)^{2}} .
$$

Step [3] does not require knowledge of the missing upper right (central, corner) BB control point $\mathbf{b}_{33}$, shown in Figure $4(b)$. Only the first three layers of the refined control net need to be derived. We call the collection $\mathbf{b}_{i j}, 0 \leq i, j \leq 3,(i, j) \neq$ $(3,3)$ a tensor-border of depth 2.

Since Step [1] requires only enough data (position, first and second derivatives) along the boundary, adjustable speed subdivision can be applied to any quad mesh with extraordinary nodes separated by one regular node; if extraordinary nodes are not separated, one Catmull-Clark step is applied.

Combinatorially, this refinement step mimics Catmull-Clark subdivision. The weights $\beta$ and $\gamma$ are chosen within a range that yields $C^{1}$ surfaces. The specific choice of $\beta$ and $\gamma$ places the central limit point at the central limit point of Catmull-Clark subdivision (see Section 3). 
With the labels $i j$ of the input control points $\mathbf{p}^{i j}$ and the labels $k$ of the refined points $\tilde{\mathbf{p}}_{k}$ as in Figure 4, Steps [1]-[4] define the subdivision rules

$$
\tilde{\mathbf{p}}_{k}=\sum_{i j} w_{k}^{i j} \mathbf{p}^{i j}
$$

Due to the symmetry across the sector bisectrix, we have $w_{6}^{i j}=w_{4}^{j i}, w_{3}^{i j}=w_{3}^{j i}$ and $w_{7}^{i j}=w_{7}^{j i}$. Due to the symmetry across the sector partitioning ray, we have $w_{k}^{i 4}=w_{k}^{i 2}$, for $k=2,5,8$. Since we construct a rotationally symmetric algorithm, only one of the $n$ sectors needs to be considered. Then, with $e_{1}:=\sigma^{2}+\bar{\sigma}, e_{2}:=$ $\sigma^{2}+3 \bar{\sigma}$, the relevant non-zero $w_{k}^{i j}$ are given by (read: $w_{2}^{23}:=\bar{\sigma} e_{1}$ )

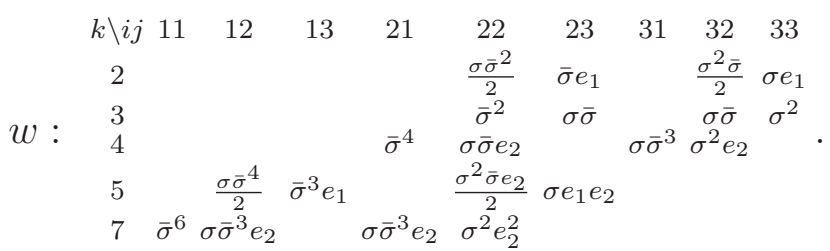

For example, for $\sigma=\bar{\sigma}=1 / 2$, as in Catmull-Clark subdivision, $\tilde{\mathbf{p}}_{3}=\left(\mathbf{p}^{22}+\mathbf{p}^{32}+\right.$ $\left.\mathbf{p}^{23}+\mathbf{p}^{33}\right) / 4$ and $\tilde{\mathbf{p}}_{2}=\left(\mathbf{p}^{22}+\mathbf{p}^{32}+6 \mathbf{p}^{23}+6 \mathbf{p}^{33}+\mathbf{p}^{24}+\mathbf{p}^{34}\right) / 16$ where $\mathbf{p}^{24}$ and $\mathrm{p}^{34}$ are two coefficients listed with the preceding sector; but unlike Catmull-Clark subdivision, $\tilde{\mathbf{p}}_{4}=\left(7 \mathbf{p}^{22}+7 \mathbf{p}^{32}+\mathbf{p}^{21}+\mathbf{p}^{31}\right) / 16$, because of the geometric knot progression. As usual, the 'outer' refined control points, corresponding to univariate non-uniform refinement with symmetry $w_{11+k}^{i j}=w_{11-k}^{j i}, k=1,2$, and

$$
w_{8}^{12}=\frac{\sigma \bar{\sigma}}{2}, w_{8}^{13}=e_{1}, w_{9}^{12}=\bar{\sigma}, w_{9}^{13}=\sigma, w_{10}^{11}=\bar{\sigma}^{3}, w_{10}^{12}=\sigma e_{2}, w_{11}^{11}=1,
$$

need not be considered for the next subdivision step. The tensor-border BB coefficients $\mathbf{b}_{i j}$ are obtained from the adjustable speed subdivision net $\mathbf{p}^{r s}$ as

$$
\mathbf{b}_{i j}:=\frac{1}{(1+\bar{\sigma})^{2}} \sum_{r s} \bar{w}_{i j}^{r s} \mathbf{p}^{r s}
$$

where the nonzero entries $\bar{w}_{i j}^{r s}$ are given in (5) unless they follow from bisectrix symmetry $\bar{w}_{i j}^{r s}=\bar{w}_{j i}^{s r}$ or sector symmetry $\bar{w}_{3 j}^{4 s}=\bar{w}_{3 j}^{2 s}$. With $d_{1}:=1+\bar{\sigma}, d_{2}:=$ $5-4 \sigma+\sigma^{2}$,

$$
\begin{array}{cccccccc}
i j \backslash r s & 11 & 21 & 31 & 22 & 32 & 23 & 33 \\
00 & \bar{\sigma}^{6} d_{1}^{2} & \sigma \bar{\sigma}^{4} d_{1} d_{2} & \sigma^{2} \bar{\sigma}^{3} d_{1} & \sigma^{2} \bar{\sigma}^{2} d_{2}^{2} & \sigma^{3} \bar{\sigma} d_{2} & & \sigma^{4} \\
10 & & 2 \bar{\sigma}^{4} d_{1} & \sigma \bar{\sigma}^{3} d_{1} & 2 \sigma \bar{\sigma}^{2} d_{2} & \sigma^{2} \bar{\sigma} d_{2} & 2 \sigma^{2} \bar{\sigma} & \sigma^{3} \\
& & \bar{\sigma}^{4} d_{1} & \bar{\sigma}^{3} d_{1} & \sigma \bar{\sigma}^{2} d_{2} & \sigma \bar{\sigma} d_{2} & \sigma^{2} \bar{\sigma} & \sigma^{2} \\
\bar{w}: & \frac{1}{2} \bar{\sigma}^{4} d_{1} & \bar{\sigma}^{3} d_{1} & \frac{1}{2} \sigma \bar{\sigma}^{2} d_{2} & \sigma \bar{\sigma} d_{2} & \frac{1}{2} \sigma^{2} \bar{\sigma} & \sigma^{2} \\
30 & & & 4 \bar{\sigma}^{2} & 2 \sigma \bar{\sigma} & & \sigma^{2} \\
11 & & & & 2 \bar{\sigma}^{2} & 2 \bar{\sigma} & \sigma \bar{\sigma} & \sigma \\
21 & & & \bar{\sigma}^{2} & 2 \bar{\sigma} & \frac{1}{2} \sigma \bar{\sigma} & \sigma \\
31 & & & \bar{\sigma}^{2} & \bar{\sigma} & & 1 \\
22 & & & \frac{1}{2} \bar{\sigma}^{2} & \bar{\sigma} & \frac{1}{2} \bar{\sigma} & 1 \\
32 & & & & & &
\end{array}
$$

The BB coefficients of Figure 4(c) defined by $\mathbf{p}^{i j}$, together with the outer layer of $\mathrm{BB}$ coefficients defined by the refined net $\tilde{\mathbf{p}}_{k}$ form a bi-3 surface ring in BB form. 

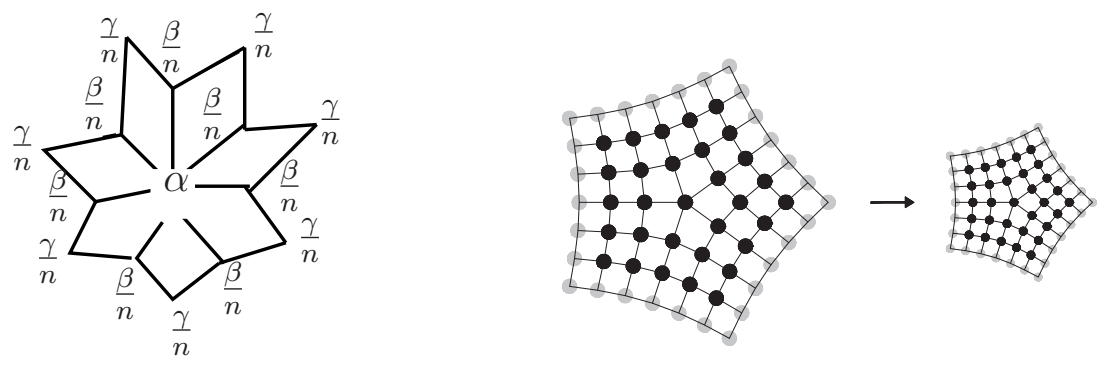

Fig. 5. (left) Rule at the central point; here $\alpha:=1-\beta-\gamma$. (right) Control net refined by adjustable speed subdivision: the black points determine the subdivision matrix $A$; black and grey points define a complete bi-3 surface ring.

One sector of this ring is shown in Figure $4(d)$ with the contribution drawn from $\mathbf{p}^{i j}$ in black, and the contribution from $\tilde{\mathbf{p}}_{k}$ in grey.

\section{Analysis of adjustable speed subdivision}

Indexing the input mesh $\mathbf{p}$ in the same manner as $\tilde{\mathbf{p}}$ (see Figure 4), we analyze the refinement

$$
\mathbf{p}_{7 i+k} \rightarrow \tilde{\mathbf{p}}_{7 i+k}:=A \mathbf{p}_{7 i+k}, \quad i=0, \ldots, n-1, k=1, \ldots, 7 .
$$

Replacing the extraordinary node by $n$ copies as in [PR98], we obtain the $7 n \times 7 n$ subdivision matrix with the familiar uniform block-circulant structure

$$
A:=\left(\begin{array}{cccc}
A_{0} & A_{1} & \ldots & A_{n-1} \\
A_{n-1} & A_{0} & \ldots & A_{n-2} \\
\vdots & & \ddots & \vdots \\
\dot{A}_{1} & \ldots & A_{n-1} & \dot{A}_{0}
\end{array}\right), \quad A_{k} \in \mathbb{R}^{7 \times 7} .
$$

The discrete Fourier Transform diagonalizes $A$ into blocks

$$
\hat{A}_{j}=\sum_{k=0}^{n-1} \omega_{n}^{j k} A_{k}, j=0, \ldots, n-1, \omega_{n}^{i}:=\exp \left(\frac{2 \pi i \sqrt{-1}}{n}\right) .
$$

The eigenvalues of the 0-th Fourier block $\hat{A}_{0}$ are: $1, \bar{\sigma}^{3}, \bar{\sigma}^{4}, \bar{\sigma}^{5}, \bar{\sigma}^{6}$ and the roots of quadratic equation

$$
\lambda^{2}+\left(\beta+\gamma-2+2 \sigma\left(\bar{\sigma}^{2}+1\right)\right) \lambda+\bar{\sigma}^{2}\left(\bar{\sigma}^{2}-(\beta+\gamma) \bar{\sigma}+\gamma \sigma\right)=0 .
$$

The eigenvalues of the $k$-th Fourier block, $\hat{A}_{k}, k \neq 0$, are $0, \bar{\sigma}^{3}, \bar{\sigma}^{4}, \bar{\sigma}^{5}, \bar{\sigma}^{6}$ and the roots of quadratic equation

$$
\lambda^{2}-\bar{\sigma}\left(\sigma^{2}\left(1+c_{n, k}\right)+2 \bar{\sigma}\right) \lambda+\bar{\sigma}^{4}=0, \quad c_{n, k}:=\cos \left(\frac{2 \pi k}{n}\right) .
$$

We determine the central limit or extraordinary point by calculating the left eigenvector to the eigenvalue 1 [HKD93]. The stencil for computing the extraordinary 
point is structurally equivalent to that of Figure 5, left. We only need to replace $\alpha$, $\beta, \gamma$ by $\breve{\alpha}:=1-\breve{\beta}-\breve{\gamma}, \breve{\beta}, \breve{\gamma}$, where

$$
\begin{aligned}
\breve{\beta} & :=\frac{2 \bar{\sigma}(\beta+\gamma)+\sigma \beta}{\left(\sigma^{2}+3 \bar{\sigma}\right) \beta+\left(2 \bar{\sigma}^{2}+\bar{\sigma}+1\right) \gamma+\sigma\left(\sigma^{2}+2 \bar{\sigma}\right)}, \\
\breve{\gamma} & :=\frac{\bar{\sigma}^{2}(\beta+2 \gamma)+\sigma \gamma}{\left(\sigma^{2}+3 \bar{\sigma}\right) \beta+\left(2 \bar{\sigma}^{2}+\bar{\sigma}+1\right) \gamma+\sigma\left(\sigma^{2}+2 \bar{\sigma}\right)} .
\end{aligned}
$$

The appendix shows how a Catmull-Clark net $\left\{\mathbf{c}^{i j}\right\}$ is transformed (scaled) to a net $\left\{\mathbf{p}_{k}\right\}$ so that adjustable speed subdivision with $\sigma=1 / 2$ and $\beta$ and $\gamma$ defined by (1) applied to $\left\{\mathbf{p}_{k}\right\}$ yields the same surface as Catmull-Clark subdivision applied to $\left\{\mathbf{c}^{i j}\right\}$. (Of course one can apply adjustable speed subdivision to $\left\{\mathbf{c}^{i j}\right\}$, but this generally results in a different surface than Catmull-Clark subdivision.)

We verify that with $c:=\cos (2 \pi / n)$ the subdominant eigenvalue is the root

$$
\lambda:=\frac{\bar{\sigma}}{2}\left((1+\mathrm{c}) \sigma^{2}+2 \bar{\sigma}+\sigma \sqrt{(1+\mathrm{c})\left((1+\mathrm{c}) \sigma^{2}+4 \bar{\sigma}\right)}\right)
$$

of the quadratic equation (8) for $k=1$. The subsubdominant eigenvalue $\mu$ is also a root of (8) but for $k=2$. As with Catmull-Clark subdivision, the limit curvature is generically unbounded since $\mu>\lambda^{2}$, and the limit surface is generically hyperbolic at the extraordinary point, the price typically paid for using simple mesh refinement rules. For any speed $\sigma \in(0,1)$, the characteristic ring is a normalized bi-3 symmetric scalable $C^{2}$ map. Due to the rotational and bisectrix symmetry only one half of one sector needs to be considered, Figure 6, left. With the normalization $\mathbf{b}_{00}:=(1,0)$, tensor-borders from adjacent sectors are $C^{2}$ connected if, with $\overline{\mathrm{c}}:=\cos (\pi / n), \overline{\mathrm{s}}:=\sin (\pi / n)$,

$$
\begin{aligned}
& \mathbf{b}_{10}:=\left(2 z_{1} \overline{\mathrm{s}}+z_{2} \overline{\mathrm{c}},-2 z_{1} \overline{\mathrm{c}}+z_{2} \overline{\mathrm{s}}\right), \mathbf{b}_{20}:=\left(z_{0} \overline{\mathrm{c}}+z_{1} \overline{\mathrm{s}}, z_{0} \overline{\mathrm{s}}-z_{1} \overline{\mathrm{c}}\right), \\
& \mathbf{b}_{30}:=\left(z_{0} \overline{\mathrm{c}}, z_{0} \overline{\mathrm{s}}\right), \mathbf{b}_{11}:=\left(2 \frac{z_{4}}{\overline{\mathrm{s}}}, 0\right), \mathbf{b}_{21}:=\left(z_{3} \overline{\mathrm{c}}+z_{4} \overline{\mathrm{s}}, z_{3} \overline{\mathrm{s}}-z_{4} \overline{\mathrm{c}}\right), \\
& \mathbf{b}_{31}:=\left(z_{3} \overline{\mathrm{c}}, z_{3} \overline{\mathrm{s}}\right), \mathbf{b}_{22}:=\left(z_{3} \overline{\mathrm{s}}-z_{4} \frac{\mathrm{c}}{2 \overline{\mathrm{s}}}, 0\right), \\
& \mathbf{b}_{32}:=\left(\overline{\mathrm{c}}\left(z_{3} \frac{1+\mathrm{c}}{2}-z_{4} \frac{\mathrm{c} \overline{\mathrm{c}}}{2 \overline{\mathrm{s}}}\right), \overline{\mathbf{s}}\left(z_{3} \frac{1+\mathrm{c}}{2}-z_{4} \frac{\mathrm{c} \overline{\mathrm{c}}}{2 \overline{\mathrm{s}}}\right)\right) .
\end{aligned}
$$

The ring defined by the scalars $z_{i}$ listed in Appendix 4.2 and its $\lambda$-scaled copy are $C^{2}$ connected. Regularity and injectivity of the characteristic ring have been checked numerically following [RP05], for $n=3,5,6, \ldots, 20$ and $\sigma \in[1 / 10,9 / 10]$ : using the $\mathrm{BB}$ representation, we formed, for two of the three $\mathrm{BB}$ patches of a sector, the differences $\mathbf{b}_{i+1, j}-\mathbf{b}_{i j}$, resp. $\mathbf{b}_{i, j+1}-\mathbf{b}_{i j}$ and verified by interval arithmetic that their coordinates have a sign pattern that implies regularity. Injectivity follows from the monotonicity of the boundary coefficients of the patch. 

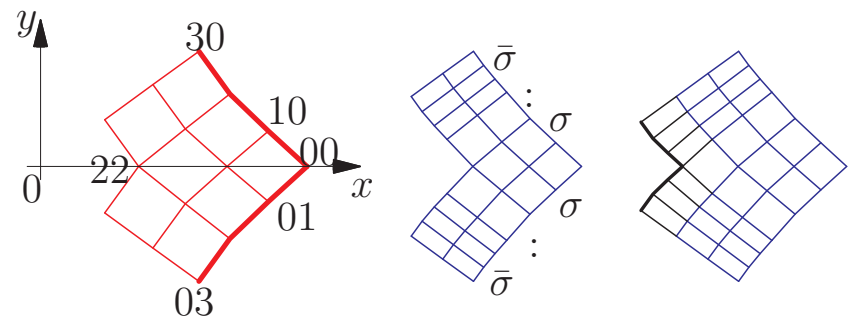

Fig. 6. Construction of one sector of the bi-3 characteristic ring. (left) The tensor-border $\left\{\mathbf{b}_{i j}\right\}$. (middle) The tensor-border is subdivided with ratio $\sigma: \bar{\sigma}$. (right) The bi-3 sector construction is completed by $\lambda\left\{\mathbf{b}_{i 0}\right\}$ and $\lambda\left\{\mathbf{b}_{0 j}\right\}$, the outermost layers of the tensor-border scaled by $\lambda$.

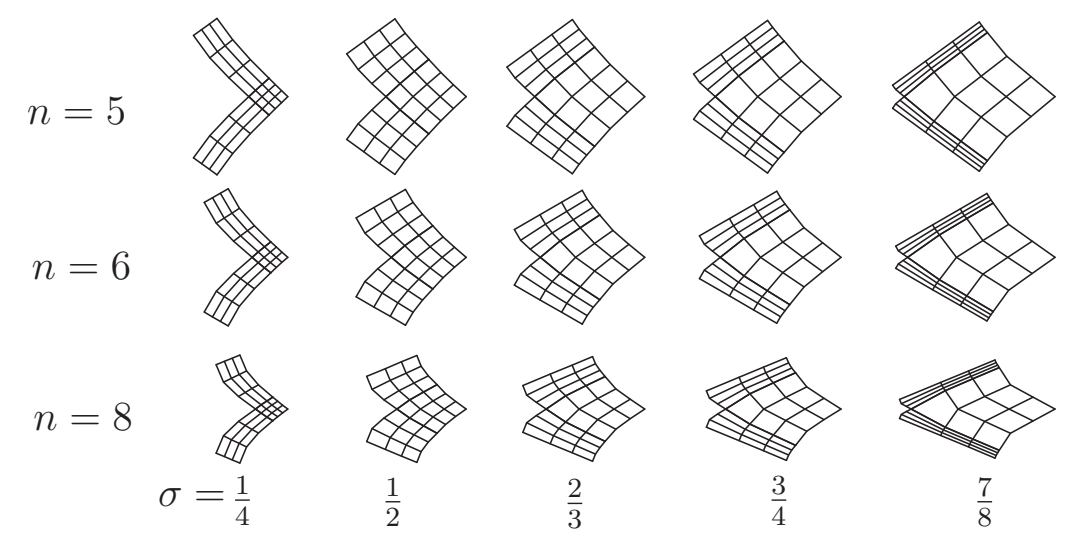

Fig. 7. A sector of the characteristic ring for different valences and speeds (see Appendix 4.2 for explicit formulas).

\section{Discussion and Applications}

Figure 7 illustrates how different choices of $\sigma$ affect the width of a sector of the characteristic ring. Figure 1 already showed corresponding variations of the width of surface rings. The surface smoothness deteriorates when $\sigma \geq 3 / 4$. This does not surprise since, in the limit as $\sigma \rightarrow 1$, we obtain the well-known construction of a finite bi-3 cap consisting of the bi-3 tensor-border plus the central limit point. However, applying two subdivision steps with $\sigma=3 / 4$ followed by one step with $\sigma=1$ confines the area lacking smoothness so it is no longer easily detectable (Figure 8).

As $e$ approaches 1, Figure 10, bottom right shows the curvature of surfaces generated by adjustable speed subdivision increasing with speed. Low speeds are not typically better than standard speeds near 1/2: Figure 10, bottom left, shows five steps of speed $\sigma=\frac{1}{4}$ yielding roughly the same result as two steps of speed $\sigma=\frac{1}{2}$. And, just as with Catmull-Clark subdivision, generically the curvature is unbounded at extraordinary points. This unboundedness does not pose a problem for using the characteristic ring as ct-map in $C^{2}$ guided subdivision [KP07b] even for high speeds (Figure 10, bottom middle, Figure 9). Here the unevenness of the ct-map is counterbalanced by stably sampling the guide surface. 

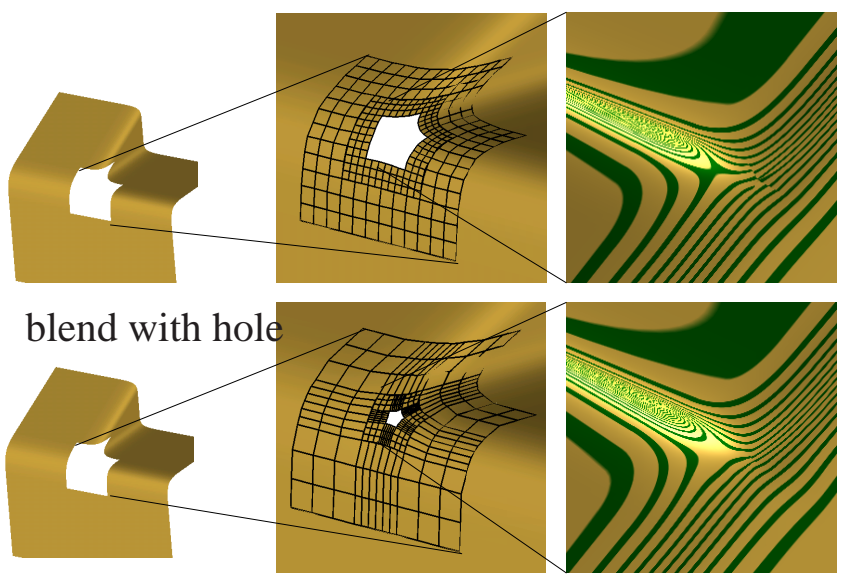

Fig. 8. Application. Hole filling using a $C^{0}$ bi-3 cap so that the lack of smoothness is not easily visible because faster subdivision confines the problem to a minimal region. (left) Blend with hole. (middle) Two steps of subdivision. (right) Highlight lines when filling the remaining hole. (top) Regular speed $\sigma=1 / 2$ exhibits the expected jump in the highlight line (top, right). (bottom) High speed $\sigma=3 / 4$ hides this fault by confining it to a smaller region.

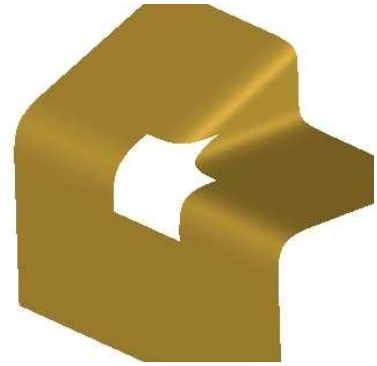

boundary data

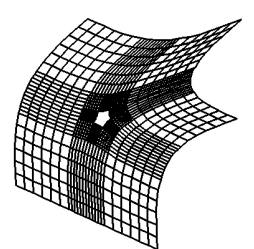

Bézier net

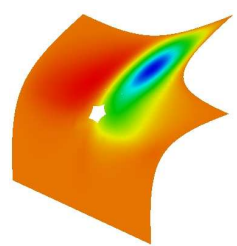

Gauss curvature

2 guided surface rings

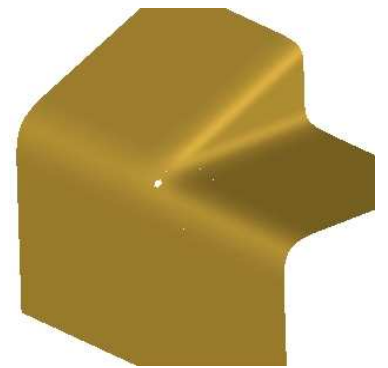

boundary +2 rings

Fig. 9. Two rings generated by guided surfacing [KP07b] using as ct-map the characteristic ring of Figure 7 with speed 3/4. The guide stabilizes the shape, counterbalancing any strong changes in parameterization.

Acknowledgement. The work supported NSF grant CCF-0728797.

\section{Appendix}

\subsection{Transformation from a Catmull-Clark net to an adjustable speed subdivision net}

To prove that adjustable speed subdivision can generate the same limit surface as Catmull-Clark subdivision, we cannot apply adjustable speed subdivision and Catmull-Clark subdivision to the same input mesh since adjustable speed subdivision assumes that its input mesh is associated with knot spacing in geometric pro- 
gression. We derive the necessary transformation between the Catmull-Clark net $\left\{\mathbf{c}^{i j}\right\}$ and the adjustable speed subdivision net $\left\{\mathbf{p}_{k}\right\}$ (see Figure 4 for the indices) by matching the $\mathrm{BB}$ representation of a Catmull-Clark net with the BB representation in Figure 4(b). Then

$$
\mathbf{p}_{k}=\sum_{i j} \dot{w}_{k}^{i j} \mathbf{c}^{i j}
$$

where nonzero entries $\dot{w}_{k}^{i j}$ are given below unless they follow from bisectrix symmetry or sector symmetry:

$$
\begin{aligned}
& f_{1}:=\bar{\sigma}-\sigma, f_{2}:=\bar{\sigma}+1, f_{3}:=2 \bar{\sigma}^{2}-1, f_{4}:=\bar{\sigma}^{3}+\bar{\sigma}^{2}-\sigma,
\end{aligned}
$$

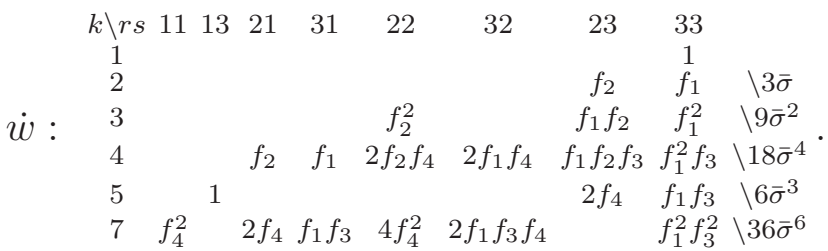

\subsection{Definition of the characteristic ring}

Here, we show all constants defining the characteristic ring (10). Representing a single set of formulas for a whole family of algorithms in terms of the speed $\sigma$ must be more complex than those of a single instance such as Catmull-Clark. For $r=0 \ldots 4$,

$$
\begin{aligned}
h_{r} & :=\frac{\sum_{i j} N U M[r][i][1][j] \mathrm{c}^{j-1}+\lambda \sum_{i j} N U M[r][i][2][j] \mathrm{c}^{j-1}}{\sum_{i j} D E N[i][1][j] \mathrm{c}^{j-1}+\lambda \sum_{i j} D E N[i][2][j] \mathrm{c}^{j-1}}, \\
z_{0} & :=\frac{h_{0}}{2 \overline{\mathrm{c}}}, z_{1}:=h_{1} \overline{\mathrm{s}}, z_{2}:=\frac{h_{2}}{2 \overline{\mathrm{c}}}, z_{3}:=\frac{h_{3}}{2 \overline{\mathrm{c}}}, z_{4}:=h_{4} \overline{\mathrm{s}},
\end{aligned}
$$

where $D E N, N U M[r], r=0 \ldots 4$ (generated by the symbolic computing tool Maple) are listed in the form

$$
[\operatorname{seq}([\operatorname{seq}([\operatorname{seq}(\mathrm{M}[\mathrm{i}][\mathrm{k}][\mathrm{j}], \mathrm{j}=1 . . \operatorname{nops}(\mathrm{M}[\mathrm{i}][\mathrm{k}])], \mathrm{k}=1 . .2)], \mathrm{i}=1 . .15)]
$$

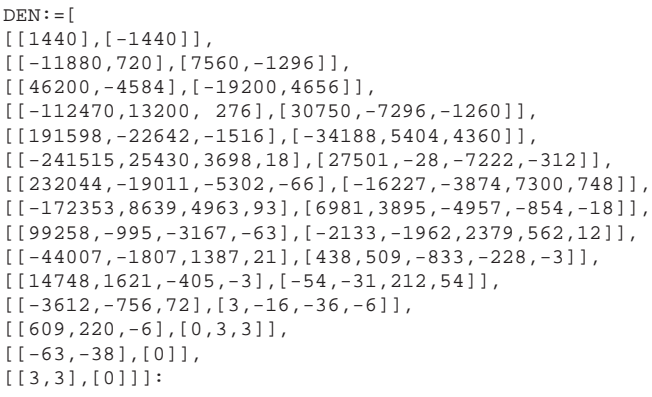

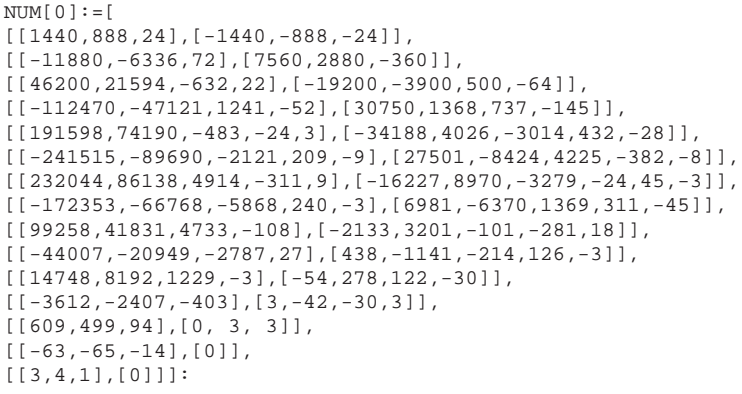


NUM $[1]:=[$

$[[480,24],[-480,-24]]$

$[[-3960,12],[2520,-252]]$,

$[[15400,-502,22],[-6400,868,-64]]$

$[[-37490,1518,-70],[10250,-761,-49]]$,

$[[63866,-1931,42,3],[-11396,-1054,422,-28]]$,

$[[-80505,479,116,-9],[9167,3535,-641,10]]$,

$[[77348,2210,-248,9],[-5409,-4539,395,33,-3]]$,

$[[-57451,-4051,219,-3],[2327,3502,-4,-42]]$,

$[[33086,3926,-105],[-711,-1752,-149,18]]$,

$[[-14669,-2556,27],[146,562,96,-3]]$,

$[[4916,1190,-3],[-18,-106,-27]]$,

$[[-1204,-400],[1,9,3]]$

$[[203,94],[0]]$

$[[-21,-14],[0]]$,

$[[1,1],[0]]]:$

NUM $[4]:=$

$[[480,80],[-480,-80]]$

$[[-3960,-390,10],[2520,-66,-18]]$

$[[15400,790,-10],[-6400,842,-94]]$

$[[-37490,-914,-81,3],[10250,-1815,219,-16]]$,

$[[63866,1003,248,-9],[-11396,1918,-92,-14]]$,

$[[-80505,-1850,-326,9],[9167,-936,-234,48,-3]]$,

$[[77348,3432,243,-3],[-5409,-170,404,-45]]$,

$[[-57451,-4531,-108],[2327,582,-305,18]]$,

$[[33086,4187,27],[-711,-415,129,-3]]$,

$[[-14669,-2772,-3],[146,158,-30]]$,

$[[4916,1326],[-18,-33,3]]$

$[[-1204,-452],[1,3]]$

$[[203,105],[0]]$,

$[[-21,-15],[0]]$,

$[[1,1],[0]]]$ :
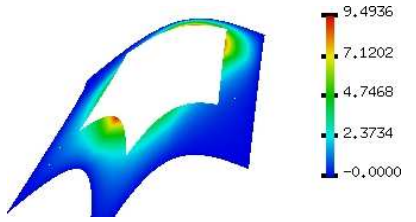

$\sigma=\frac{1}{4}$
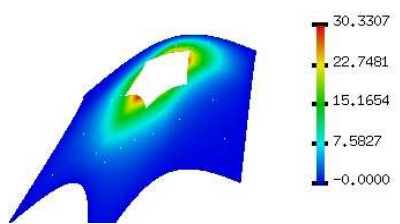

$$
\sigma=\frac{1}{4}(5 \text { rings })
$$

NUM [2] $:=[$

$[[1440,1104,48],[-1440,-1104,-48]]$.

$[[-11880,-8208,24],[7560,4176,-504]]$

$[[46200,29316,-872,44],[-19200,-7896,1352,-128]]$

$[[-112470,-67158,2468,-140],[30750,9504,-1168,-98]]$,

$[[191598,110574,-2960,102,6],[-34188,-7872,-744,676,-56]]$

$[[-241515,-138374,549,166,-18],[27501,4440,2885,-1050,20]]$

$[[-2415,135427,3722,-403,18],[-16227,-1358,-3369,752,48,6]$

$[[232044,135427,3722,-403,18],[-16227,-1358,-3369,752,48,-6]]$

$[-172353,-104991,-6721,375,-6],[6981,-283,2185,-199,-72]]$,

$[[99258,64597,6662,-189],[-2133,640,-772,-88,33]]$

$[[-44007,-31310,-4500,51],[438,-412,67,87,-6]]$,

$[[14748,11747,2185,-6],[-54,152,59,-27]]$,

$[[-3612,-3304,-764],[3,-32,-24,3]]$,

$[[609,658,185],[0,3,3]]$,

$[[-63,-83,-28],[0]]$,

$[[3,5,2],[0]]]:$

NUM $[3]:=[$

$[[960,856,88],[-960,-856,-88]]$

$[[-7920,-6462,-356,10],[5040,3414,-204,-18]]$,

$[[30800,23352,532,-4],[-12800,-6822,952,-114]]$,

$[[-74980,-53860,-334,-99,3],[20500,8756,-1467,173,-16]]$

$[[127732,88788,315,266,-9],[-22792,-7920,1006,10,-20]]$,

$[[-161010,-110639,-1358,-332,9],[18334,5283,82,-324,48,-3]]$,

$[[154696,107286,3216,243,-3],[-10818,-2669,-834,440,-45]]$,

$[[-114902,-82039,-4477,-108],[4654,1041,846,-311,18]]$,

$[[66172,49570,4181,27],[-1422,-317,-475,129,-3]]$,

$[[-29338,-23483,-2772,-3],[292,74,164,-30]]$,

$[[9832,8562,1326],[-36,-12,-33,3]]$,

$[[-2408,-2324,-452],[2,1,3]]$

$[[406,443,105],[0]]$

$[[406,443,105],[0]]$

$[[2,3,1],[0]]]:$

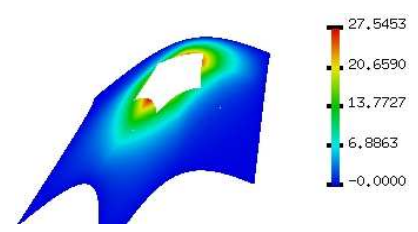

$\sigma=\frac{1}{2}$

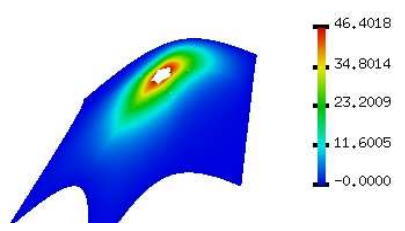

$\sigma=\frac{3}{4}$ (guided)

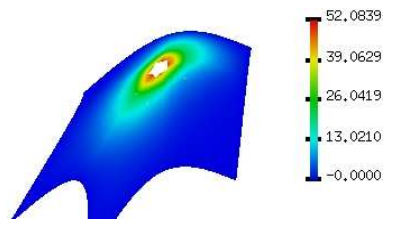

$\sigma=\frac{3}{4}$

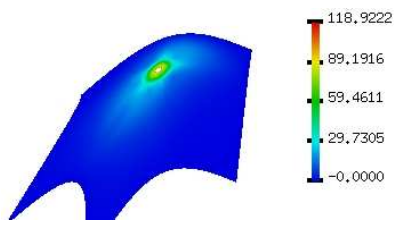

$\sigma=\frac{7}{8}$

Fig. 10. Gauss curvature of two rings (except bottom left, five rings) generated by adjustable speed subdivision (except bottom middle, where guided subdivision is applied) for the example of Figure 1. The maximal curvatures are (top) 9.5, 27.5, 52, (bottom) 30, 46.5, 119.

\section{References}

[BHR93] Carl de Boor, K. Höllig, and S. Riemenschneider. Box splines. Springer-Verlag New York, Inc., New York, NY, USA, 1993.

[CDS07] T. J. Cashman, N. A. Dodgson, and Malcolm A. Sabin. Non-uniform B-spline subdivision using refine and smooth. In Proceedings Mathematics of Surfaces 12, pages 121-137, 2007.

[CDS08] T. J. Cashman, N. A. Dodgson, and Malcolm A. Sabin. A symmetric, nonuniform, refine and smooth subdivision algorithm for general degree B-splines. 
Computer Aided Geometric Design, pages 121-137, 2008.

[CLR80] E. Cohen, T. Lyche, and R. Riesenfeld. Discrete B-splines and subdivision techniques in computer-aided geometric design and computer graphics. Comput. Gr. Image Process., 14:87-111, October 1980.

[GW93] Ron Goldman and Joe D. Warren. An extension of Chaiken's algorithm to Bspline curves with knots in geometric progression. CVGIP: Graphical Model and Image Processing, 55(1):58-62, 1993.

[HKD93] Mark Halstead, Michael Kass, and Tony DeRose. Efficient, fair interpolation using Catmull-Clark surfaces. In James T. Kajiya, editor, Computer Graphics (SIGGRAPH '93 Proceedings), volume 27, pages 35-44, August 1993.

[KP07a] K. Karčiauskas and J. Peters. Adjustable speed subdivision surfaces. Technical report, University of Florida CISE, TR-07-430, 2007.

[KP07b] K. Karčiauskas and J. Peters. Concentric tesselation maps and curvature continuous guided surfaces. Computer-Aided Geometric Design, 24(2):99-111, Feb 2007.

[MRF06] K. Müller, L. Reusche, and D. Fellner. Extended subdivision surfaces: Building a bridge between NURBS and Catmull-Clark surfaces. ACM Transactions on Graphics, 25(2):268292, April 2006.

[PR98] J. Peters and U. Reif. Analysis of generalized B-spline subdivision algorithms. SIAM Journal on Numerical Analysis, 35(2):728-748, April 1998.

[RP05] U. Reif and J. Peters. Topics in multivariate approximation and interpolation. In K. Jetter et al., editor, Structural Analysis of Subdivision Surfaces - A Summary, pages 149-190, 2005.

[SG08] S. Schaefer and R. Goldman. Non-uniform subdivision for B-splines of arbitrary degree. Computer Aided Geometric Design, pages 121-137, 2008.

[SZBN03] Thomas W. Sederberg, Jianmin Zheng, A Bakenov, and A. Nasri. T-splines and T-NURCCS. ACM Transactions on Graphics, Siggraph 2003, page 477-484, 2003.

[SZSS98] Thomas W. Sederberg, Jianmin Zheng, David Sewell, and Malcolm A. Sabin. Non-uniform recursive subdivision surfaces. In SIGGRAPH, pages 387-394, 1998. 